\title{
Preparation and characterization of polymers imprinted molecularly with ibuprofen and nitrobenzene
}

\author{
(INDEX: 117-124/2010 Copernican Letters ${ }^{\circledR}{ }^{\circ o l} 1$ ) \\ Bartlomiej Wasiniak, Jerzy P. Lukaszewicz \\ Department of Fundamentals of Chemistry \\ Research Group for Modeling and Synthesis of Novel Materials \\ Faculty of Chemistry,Nicolaus Copernicus University 7 Gagarin Str., PL-87100 Torun \\ (Poland) \\ bartas.83@interia.pl
}

\begin{abstract}
In this work molecularly imprinted polymer (MIP) which can be later used for SPME improvement were prepared. Polymerization reaction was initiated in six different ways and for this purpose six different solvents as porogens were used. Prepared MIP's were stable in temperature range $160-200^{\circ} \mathrm{C}$. Pore sizes and BET surface were characterized by sorption of nitrogen at liquid nitrogen temperature. On the contrary to most MIPs, the studied MIPs were strictly nanoporous i.e. most of total pore volume was attributed to pores ranging from 3 to $1.0 \mathrm{~nm}$ in diameter. BET surface area exceeded even $300 \mathrm{~m}^{2} / \mathrm{g}$. Basic conditions of the MIPs synthesis have been optimized with particular respect to type of solvent and polymerization initiator which are basic factors influencing the ability of the MIP towards adsorption of ibuprofen/nitrobenzene.
\end{abstract}

\section{Introduction}

In present years it has been popular development of different materials which have strongly defined properties. Examples of such materials are new liquid crystals [1], sensing materials for explosives detection [2], conducting polymers [3], polymers for pharmaceutical applications [4] and intelligent very selective polymers so called Molecularly Imprinted Polymers (MIP's) [5]. Application of these materials is wide and covers before all chemical analysis (as a sorbent for solid-phase extraction), enzymatic catalysis, membrane separation, etc. MIP's are particularly suitable for SPME fibre coating because of high selectivity, chemical stability and controlled preparation. MIP's by nature should be porous and the cavities in the solid should reflect the size and chemical structure of molecules used for imprinting. Therefore the size of pores should be similar to the size of molecules. MIP's are prepared in process called as molecular imprinting.

Molecular imprinting is a technique of preparation chemically selective binding sites, which recognize particular molecules by their adsorption in the cavities in polymer matrix [6]. Molecular imprinting technology involves the preparation of a polymer with specific recognition sites for certain molecules. Once the polymer has been obtained, it can be used in solid-phase extraction protocols, where a careful selection of the most appropriate solvents to be used in the different steps (sample loading, washing and elution) is needed in order to extract the target analyte selectively. The concept of exploitation of molecular interactions in the system template molecule-solid matrix is rather old (late 19th century first announcements appear). One of the most important events was that in 1984 Emil Fisher has presented a "lock-and-key" metaphor concerning the way a substrate interact with the enzyme. This has become the fundament of supramolecular chemistry [10]. MIP can be defined as special kind of polymer matrix in which template molecule has a strongly defined position during polymerization of matrix-forming monomer and movements of molecule are frozen in desired configuration [11]. This causes the formation of recognition sites in the matrix selective for the template after the removal 
of template. Template can be washed by using different solvents. The template can be a small molecule before all like drugs, amino acids, steroid hormones [7]. There are two general approaches in molecular imprinting. First is covalent approach, where template-monomer is bonded in solution prior to polymerization due to reversible, covalent bounds. Molecular recognition between the polymers is dependent of the formation of these bonds [10]. Second is noncovalent approach. In this approach prearrangement between the template and monomer is formed by noncovalent or coordination interactions. Although it can be applied for almost all type of templates, result of polymer preparation using this approach is more difficult to control. Interactions between template and the functional monomer molecule are in this case weaker. In a consequence the binding sites formed in a polymer matrix are very heterogenic [6,7]. This approach is also very sensitive to the polarity of the solvent, which is a consequence of noncovalent interactions employed [6]. Literature quotes numerous MIP's to be porous [7-9]. However, in many cases there is a lack of experimentally verified information on pore structure of MIPs, like BET surface area and pore size distribution (PSD function). A closer look on some research lets to disclosed that most of described MIP's are mesoporous and macroporous solids, according to the IUPAC classification, in which the size of cavities is several tens to hundreds bigger than the known dimensions of template molecules. However, the comparativeness of template molecules and pores sizes according to authors is essential to call the polymers "molecularly" imprinted. The aim of this research to prepare a strictly nanoporous non-imprinted polymers and polymers imprinted by ibuprofen and nitrobenzene with high BET surface.

\section{Experimental}

\subsection{Chemicals \& Reagents}

All chemicals and reagents used were of analytical reagent grade with no further purification. Methacrylic acid, ethylene glycol dimethacrylate, n-hexane, chloroform, dichloromethane (analytical reagent grade), (Sigma-Aldrich, Germany); acetonitrile, methanol gradient-grade (Baker, Deventer, Holland). Ibuprofen, nitrobenzene were purchased from SigmaAldrich (St. Louis, MO, USA).

\subsection{Preparation of polymer coatings}

Firstly, several polymer materials were synthesized using different porogens and methods of initiation of polymerization. Among many factors that influence synthesis, the most important is template molecule. The availability and type of the functional groups in the template molecule play important role. Also the structure, solubility of the template and the end-use of imprinted material will decide about imprinting method [12]. For this experiment non-covalent approach of molecular imprinting was applied. Templates used during the polymerization were ibuprofen as one of the most common no steroid anti-inflammatory drug and nitrobenzene as one of substances classified as explosives. Both of these compounds are soluble in organic solvents as chloroform or acetonitrile and their molecules contain groups that are able to create hydrogen bounding. These make them applicable for molecular imprinting process.

\subsection{1 . Choosing a functional monomer}

The most important is fact that functional monomer should be complementary with template molecule for creating stable prepolimerization complex and further molecular imprinting. The probability of "working properly" molecular imprinting is increasing with increasing number of independent groups 
interacting with ready polymer matrix and functional monomer [13]. Molecule of functional monomer should have functional groups that are able to create weak bonds with template regarding a further removal of the template. It can be ensured basing on acid/base properties of functional monomer. The most common in used acid monomers are: acrylic acid, methacrylic acid or 4-vinylbenzoic acid [15]. Carboxylic groups attract other molecules and donor or acceptor hydrogen bonds or by dipoledipole affecting create an ion-pair [7-9]. In this study methacrylic acid played the role of functional monomer.

\subsubsection{Crosslinker}

Crosslinker should make the polymer structure flexible to ensure free access of template molecule to the recognition sites and/or their elution. The mostly used crosslinkers are divinylbenzene or ethylene glycol dimethacrylate [10]. In this research ethylene glycol dimethacrylate was selected because it suits to the functional monomer molecule and is able to generate materials with adequate mechanical stability.

\subsubsection{Porogen}

Porogen limits the strength of interaction between template molecules and functional monomer. Generally with rising of porogen polarity the strength of recognition effect is decreasing [17]. Methanol, chloroform or dimethylforamide are commonly used as porogen. For MIP's there is defined selective or specific capture of template molecule. Specific capture means that molecularly imprinted polymer is able to adsorb substance from unary solution. Selective capture means that polymer will adsorb substance present in multicomponent mixture [15]. Authors selected acetonitrile as porogen since it creates sponge-like structure of MIP's and helps create micro pores $[6,7,10]$.

\subsubsection{Choosing an appropriate synthesis conditions}

During this part of the experiment 30 different polymers were fabricated. First Non Molecularly Imprinted Polymers (NMIP) were synthesized. NMIP's were prepared using the same amount of functional monomers $86.09 \mathrm{mg}$ ( $1 \mathrm{mmol} 0,74$ $\mathrm{ml})$ of methacrylic acid and $5 \mathrm{mmol}(1,041$ $\mathrm{ml}$ ) of ethylene glycol dimethacrylate. Six solvents were applied in quantity of $10.0 \mathrm{ml}$ as porogen: DMF, chloroform, dichloromethane, n-hexane, acetonitrile and chloroform/dichloromethane. The solvents were added in the volumetric proportion 1:1 regarding the total volume of the above listed reagents. Polymerization was initiated alternatively by benzoil peroxide (BPO), 2,2'-azobis-isobutyronitrile (AIBN), ammonium persulfate, hydrogen peroxide and $\gamma$ - radiation. After addition of $100 \mathrm{mg}$ of AIBN or $220 \mathrm{mg}$ of BPO or $500 \mathrm{mg}$ of $\left(\mathrm{NH}_{4}\right)_{2} \mathrm{~S}_{2} \mathrm{O}_{8}$ or $2.5 \mathrm{ml}$ of $\mathrm{H}_{2} \mathrm{O}_{2}$ samples were mixed carefully by a magnetic stirrer (1000 rpm for 5 minutes). In last case, polymerization was initiated by $\gamma$ - radiation. After mixing samples were frozen in liquid nitrogen, then closed in glass ampoules and subjected to $\gamma$ - radiation for 24,48 and 72 hours. When polymerization finished glass ampoules were smashed, polymer materials milled until grain size $40-75 \mu \mathrm{m}$ and washed from the rest of reactants using chloroform, toluene and then acetone. After drying in vacuum for 72 hours samples were taken for further analysis. In this way NMIP's preparation conditions were tested. In the second stage, following this synthesis procedure, MIP's were fabricated by adding ibuprofen or nitrobenzene as templates. Also these samples were milled, dried and washed. Although thermal stability of prepared NMIP's and MIPS was good (mass loss below $5 \%$ up to $150^{\circ} \mathrm{C}$ ), BET surface areas were very low for prepared MIP's. (i.e. $4.66 \mathrm{~m}^{2} / \mathrm{g}$ ). That forced a change of polymerization conditions (proportions, 
temperature, duration, solvent). Basing on literature announcement [19] the synthesis declined to the following pathway:

In reaction flask $4.72 \mathrm{ml}$ EGDMA was added to $0.28 \mathrm{ml}$ of MAA as functional monomer and mixed with $10.0 \mathrm{ml}$ of acetonitrile. Then $300 \mathrm{mg}$ of AIBN as reaction polymerization initiator was added. At the end appropriate amount of template (ibuprofen/nitrobenzene) was putted into reaction flask. All components were mixed by using magnetic stirrer and after sonification (30 minutes) reaction mixture was kept on magnetic stirrer with heating in $50{ }^{\circ} \mathrm{C}$ for 24 hours. After this step called prearrangement, temperature of mixture was raised until $80^{\circ} \mathrm{C}$ and after 3 hours mixture became a white solid what meant that polymerization process was finished. At the end glass ampoules were smashed, polymer materials milled until grain size 40-75 $\mu \mathrm{m}$ and washed from the rest of reactants using chloroform, toluene and then acetone.

\subsection{Thermal analysis}

Thermal properties of the samples were determined using a thermoanalyzer TGA-DTA Thermal Analysis SDT 2960 (TA Instruments). Samples of 2 up to $15 \mathrm{mg}$ were accurately weighed into aluminum pans and then sealed. The samples were tested under a nitrogen atmosphere at a heating rate of $5^{\circ} \mathrm{C} / \mathrm{min}$ at a temperature range of $20^{\circ} \mathrm{C}$ to $550^{\circ} \mathrm{C}$.

\subsection{BET-surface area analysis}

Adsorption of nitrogen in liquid nitrogen temperature is suitable method than give possibility of calculation of BET surface area and information about pore size distribution in the sample. Adsorption of nitrogen was done using GEMINI VI Surface and Pore Analyzer (Micrometrics) and ASAP 2010 (Micromeritics Instrument Corp., Nocross, USA) porozymeter. Sample of $0.01 \mathrm{~g}$ up to $0.05 \mathrm{~g}$ was accurately weighed in tube and nitrogen adsorption/desorption experiment was done in range 0.0 to 1.0 relative pressure $\left(\mathrm{P} / \mathrm{P}_{0}\right)$ range.

\subsection{Scanning electron microscopy}

Scanning electron microscopy was used to study the morphology of prepared polymeric materials. Samples were coated with gold palladium for 70 seconds under an argon atmosphere using a Pelco Model 3 cold sputter module (TED Pella, Tustin, CA) in a high-vacuum evaporator equipped with an omni-rotary stage. Scanning electron microscopy was performed using Leo model 1530 scanning electron microscope (Zeiss/Leo (Oberkochen, Germany) at 2.5 to $1.0 \mathrm{kV}$.

\subsection{IR spectroscopy analysis}

The infrared spectra of template molecules and polymer matrix were done using Spectrum 400 FT-IR/FT-NIR spectrophotometer (Perkin Elmer, Inc. Waltham, USA). Solid sample was putted into the contact window (ATR method) and scanned from 400 up to $400 \mathrm{~cm}^{-1}$, with 10 scans.

\section{Results and discussion}

Polymerization process can be described by the scheme (see figure 1).

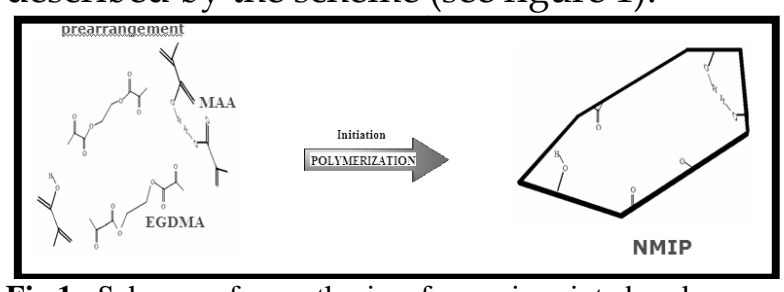

Fig.1. Scheme of synthesis of non imprinted polymer (NMIP)

The first step is so called prearrangement in which weak interactions between all the reactants taken to polymerization make all the molecules organized in reaction mixture on the specific way. Probably at this stage weak hydrogen bonds or ion-pair interactions are dominating in this process [7-9]. After initiation a stable polymer structure is being created. It is quite probable that ibuprofen/nitrobenzene can 
be captured into polymer matrix by weak hydrogen bonds between ibuprofen/nitrobenzene molecule and the functional monomer.

\subsection{Characterization of the polymers}

As mentioned, in first part of the experiment six organic solvents were used for preparation of different polymer materials (NMIP's and MIP's). In this way we were trying to find how porogen and reaction initiation influence the structure of synthesized material. In each experiment polymer mass, its density and volume were different except experiments with gamma radiation for initiation. There weren't significant differences in physical properties of prepared samples with increasing time of exposition sample to the $\gamma$ rays' source.

Thermal analysis reveal that thermal stability of samples made in different solvents using different methods of initiation of polymerization reaction are also different. The most thermal stable were polymers made using DMF and acetonitrile as solvent.

The lowest stability was obtained for polymer synthesized in chloroform or dichloromethane. In these two cases about $20 \%$ of polymer mass is lost in temperature in range $250-300^{\circ} \mathrm{C}$. The lowest mass losses were when BPO or AIBN were polymerization initiator. It can be said that the lowest thermally stable material was polymer prepared using $\gamma$ - radiation for reaction initiation (loss about $40 \%$ of mass in $300^{\circ} \mathrm{C}$ ). Although polymers prepared using $\gamma$ - radiation were as not as stable, as those when chemical initiation was applied, this method of reaction initiation needs to be investigated further because of its novelty. Physical parameters of materials prepared in first part of experiment are shown in Table 1.
Table 1.

BET surface area $\left(\mathrm{m}^{2} / \mathrm{g}\right)$ measured for prepared NMIP's

\begin{tabular}{|l|l|l|l|l|l|}
\hline \multicolumn{1}{|c|}{} & \multicolumn{5}{|l|}{ Metod of polymerization initiation } \\
\hline Type of solvent & $\gamma$ & BPO & AIBN & $\mathrm{H}_{2} \mathrm{O}_{2}$ & $\left(\mathrm{NH}_{4}\right) \mathrm{S}_{2} \mathrm{O}_{8}$ \\
\hline Dichloromethane & 2.18 & 16.22 & 8.50 & 2.64 & 3.48 \\
\hline Hexane & 3.42 & 15.25 & 3.66 & 3.13 & 2.09 \\
\hline Acetonitrile & 4.66 & 36.37 & 10.95 & 37.15 & 1.87 \\
\hline Chloroform & 1.94 & 16.42 & 7.82 & 1.06 & 0.75 \\
\hline DMF & 0.60 & $\mathrm{~nm}$ & $\mathrm{~nm}$ & $\mathrm{~nm}$ & 1.05 \\
\hline $\begin{array}{l}\text { Dichloromethane } \\
\text { Chloroform (1:1) }\end{array}$ & 4.37 & 10.16 & $\mathrm{~nm}$ & 0.25 & 2.11 \\
\hline
\end{tabular}

It can be generally said that the lowest thermal stability was observed for samples prepared with hydrogen peroxide as initiator of polymerization reaction (see Table 1). It was in range $46-151^{\circ} \mathrm{C}$. The highest temperatures in which polymer mass loss is lower than 5\% were observed for samples prepared using chemical initiators of polymerization (AIBN, BPO). It is easily to find in literature information about thermal stability of molecularly imprinted polymers. J. Svenson and co. [18] were checking thermal stability of

methacrylic acid-ethylene glycol dimethacrylate co-polimers. They have study changes in theophyline recognition characteristics of this and a non-imprinted, reference polymer arising from exposure to elevated temperatures and a range of chemical environments. They proofed that polymers can be exposure to the temperatures of up to $150^{\circ} \mathrm{C}$ (for 24h) without loss of affinity to the template.

Unfortunately BET surface area measured for these all samples was not satisfactory (ca. $2-40 \mathrm{~m}^{2} / \mathrm{g}$ ). In the another part of the experiment a set of different polymer materials were prepared using three solvents (acetonitrile, hexane,chloroform), 3 different methods of polymerization initiation (adding BPO, AIBN and affecting of $\gamma$ - radiation to the sample) and $50 \mathrm{mg}$ ibuprofen as a template molecule. In this case nine different materials were prepared. Thermal stability of these materials were satisfactory, from 98 up to $258^{\circ} \mathrm{C}$, but measured BET surface was only $1-23 \mathrm{~m}^{2} / \mathrm{g}$. 
Because of this fact another changes in the procedure of synthesis molecularly imprinted materials were necessary. In this case time of reaction was longer and all polymerization process was carried in two steps. At the beginning, after preparing reaction mixture, it was kept on magnetic stirrer in $50^{\circ} \mathrm{C}$ for 24 hours to let prearrangement step be finished. After that temperature of reactive vessel was raised up to $80^{\circ} \mathrm{C}$ and was kept for 3 hours until reaction was finished. (white solid

material was observed). On this way samples imprinted with different amount of ibuprofen and nitrobenzene were prepared. Tables 2 and 3 present BET surface area measured for prepared polymers.

Table 2 - 3

BET surface area $\left(\mathrm{m}^{2} / \mathrm{g}\right)$ for polymers imprinted with different amount of ibuprofen and nitrobenzene

\begin{tabular}{|l|l|}
\hline $\begin{array}{l}\text { Amount of } \\
\text { nitrobenzene }\end{array}$ & $\begin{array}{l}\text { BET surface } \\
\text { area }\left[\mathrm{m}^{2} / \mathrm{g}\right]\end{array}$ \\
\hline $\begin{array}{l}0.3 \mathrm{ml}[2.92 \\
\mathrm{mmol}]\end{array}$ & 363 \\
\hline
\end{tabular}

\begin{tabular}{|l|l|}
\hline $\begin{array}{l}\text { Amount } \\
\text { of } \\
\text { ibuprofen } \\
{[\mathrm{mg}]}\end{array}$ & $\begin{array}{l}\text { BET } \\
\text { surface } \\
\text { area } \\
{\left[\mathrm{m}^{2} / \mathrm{g}\right]}\end{array}$ \\
\hline 50 & 254 \\
\hline
\end{tabular}

In these cases BET surface area measured was in range $250-390 \mathrm{~m}^{2} / \mathrm{g}$. These polymers were stable in temperatures range 150$250^{\circ} \mathrm{C}$. It should be said that polymers with such high BET surface area are not common in literature. In most cases it is in range 2-50 $\mathrm{m}^{2} / \mathrm{g}$. G. Bunte at al. [19] presented very similar method of synthesis molecularly imprinted polymers using MAA, EGDMA as functional and cross linking monomers. Polymerization was chemically initiated by adding of AIBN. Although this group achieved molecularly imprinted materials (2,4,6- TNT and 2,4 -DNT were template molecules) with high BET surface area (in range $200-400 \mathrm{~m}^{2} / \mathrm{g}$ ) the average pore size was about $40-50 \AA$. The aim of this study was to prepare microporous polymer imprinted with nitrobenzene and ibuprofen. It was achieved during described syntheses. Adsorption of nitrogen done in liquid nitrogen temperature gave information about pore size. For typical pore size distribution for polymer prepared using $10 \mathrm{mmol}$ of nitrobenzene as template the maximum of the curve is about 4-5 $\AA$.

Obtained this way prepared NMIP's and MIP's imprinted with ibuprofen/nitrobenzene were characterised by the means of FTIR spectroscopy. Typical FTIR spectra of NMIPs (line "a") and nitrobenzene-MIP (line " $b$ ") is shown in Fig 2.

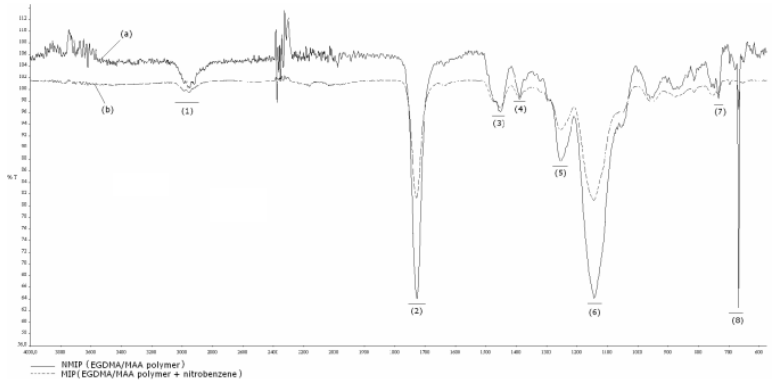

Fig. 2. FTIR spectrum of NMIP and MIP imprinted by nitrobenzene

On the spectra can notice a broad band (1) at $\bar{v}=3108-3078 \mathrm{~cm}^{-1}$ which can be assigned to the $\mathrm{C}-\mathrm{H}$ stretching vibrations of $\mathrm{CH}_{2}=\mathrm{CH}[20,21]$ from MAA and EGDMA molecules when non imprinted material spectrum is being discussed (line "a" figure 2). It can be caused by O-H valence vibrations of hydroxyl group present in MAA molecule. The intensive singlet (2) at about $\bar{v}=1725 \mathrm{~cm}^{-1}$ is due to the stretching vibrations of the carboxyl groups $\mathrm{C}=\mathrm{O}$ presented in functional monomer and cross linker molecules. Signal (3) at $\bar{v}=1466-1479 \mathrm{~cm}^{-1}$ is a typical band for $\mathrm{C}=\mathrm{O}$ stretching vibrations or $\mathrm{C}-\mathrm{H}$ deformating vibrations from methyl groups presented in cross linker molecule [20]. The band (4) at $\bar{v}=1347-1363 \mathrm{~cm}^{-1}$ corresponds to $\mathrm{C}=\mathrm{O}$ stretching vibrations in EGDMA molecule or $\mathrm{C}-\mathrm{H}$ deformating vibrations from methyl groups presented in cross linker molecule [18]. Band (5) about $\bar{v}$ $=1247 \mathrm{~cm}^{-1}$ is due to $\mathrm{C}=\mathrm{O}$ stretching vibrations. Strong signal (6) above $\bar{v}=$ $1108-1163 \mathrm{~cm}^{-1}$ is caused by $\mathrm{C}-\mathrm{H}$ 
deformating vibrations [18]. Signal

above $\bar{v}=750 \mathrm{~cm}^{-1}$ is due to out of plane C$\mathrm{H}$ deformating bonds. An intensive signal at $\bar{v}=650 \mathrm{~cm}^{-1}$ corresponds to $\mathrm{C}-\mathrm{H}$ deformating vibrations of $\mathrm{CH}_{2}=\mathrm{CH}$ from MAA and EGDMA. FTIR spectrum of polymer imprinted by nitrobenzene (line " $b$ " figure 2) show changes in the intensivity of four peaks. Signal (1) at $\bar{v}=3108-3078$ $\mathrm{cm}^{-1}$ corresponded to $\mathrm{C}-\mathrm{H}$ stretching vibrations of $\mathrm{CH}_{2}=\mathrm{CH}$ from MAA and EGDMA molecules, can be also caused by aromatic $\mathrm{C}-\mathrm{H}$ vibrations in nitrobenzene aromatic ring or asymmetric $\mathrm{NO}$ stretch vibrations. This is the reason why intensivity of this band is higher. Decrease of intensivity of signal (2) about $\bar{v}=1725$ $\mathrm{cm}^{-1}$ when IR spectrum of imprinted material is being considered, can be result of blocking of the carboxyl groups $\mathrm{C}=\mathrm{O}$ presented in functional monomer molecules by nitrobenzene [22-23]. Analogous situation is observed about $\bar{v}=1247 \mathrm{~cm}^{-1}$ signal (5). Decreasing intensivity is probably caused by the same reason. Also decreasing of signal (6) above $\bar{v}=1108$ $1163 \mathrm{~cm}^{-1}$ is caused by blocking $\mathrm{C}-\mathrm{H}$ deformating vibrations in functional monomer/cross linker molecules [23]. When IR spectrum of imprinted polymer is being considered, signal (4) at $\bar{v}=1347$ $1363 \mathrm{~cm}^{-1}$ corresponded to $\mathrm{C}=\mathrm{O}$ stretching vibrations and $\mathrm{C}-\mathrm{H}$ deformating vibrations from methyl groups presented in cross linker molecule can be also caused by C-N and $\mathrm{N}=\mathrm{O}$ stretching vibrations from nitrobenzene molecule [24]. It is not so easy to explain because of covering (simple adding) of these bands.

Scanning electron micrograph of the NMIP surface is shown on figure 3 .

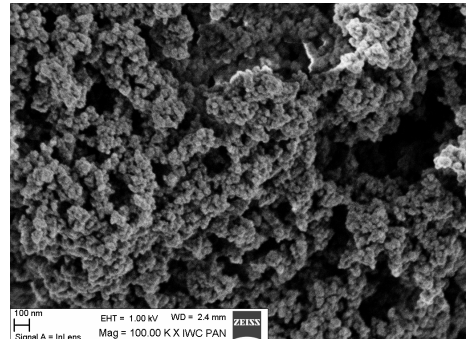

Fig. 3. Scanning electronmicrograph of the nonimprinted polymer surface. Funtional monomer - MAA, crosslinker - EGDMA, porogen - hexane

Magnification $100 \mathrm{kX}$ shows that there is possibility to have homogenous surface of the coating. There are a lot of pores with diameter in the polymer (about 5-10 nm). The highest homogeneity was achieved by the polymerization when DMF, acetonitrile, hexane with AIBN and BPO were used. In these cases we achieved homogenous surface of coating with pores in range 2.5-15 $\mathrm{nm}$.

\section{Conclusions}

Polymerization process and the imprinting process can be described by scheme (see figure 4).

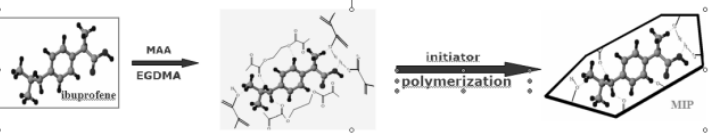

Fig. 4. Scheme of the molecular imprinting process using ibuprofen as a template

After prearrangement of a template, functional monomer, crosslinker and adding of the initiator, stable polymer matrix with recognition sites is being created (figure 4). The most probably mechanism of affecting between ibuprofen molecules and functional monomers is by hydrogen bonds. That is proper with the theory of no covalent imprinting. It is caused probably by carboxylic groups which can affect with other molecules as donor or acceptor of hydrogen bonds or by dipole-dipole affecting create an ion-pair. According to the polymerization conditions properties of polymer such like BET surface or pores size are different. Although prepared materials are stable in the range 
$200-250^{\circ} \mathrm{C}$. The best thermal stability was achieved for acetonitrile or dichloromethane when BPO was an initiator of polymerization.

The results of this study demonstrate that the prepared polymers imprinted with ibuprofen (or other drugs) can be potentially useful for SPME improvement because of their stability, simplicity of preparation and structure of polymer coating.

\section{LITERATURE}

[1] J.-D. Marty et al. The European Physical journal E 17. (2005) 515-520.

[2] A. Mc Cluskey, Organic \& biomelcular Chemistry, 2007, 5, 3233-3244.

[3] K. Prasad, Sensors and Actuators B 123 (2007), 65-70

[4] C. Donini et al. International Journal of Pharmaceutics 245 (2002) 83-91.

[5] H. Yan, International journal of molecular Sciences 2006, 7 155-178.

[6] M. Komiyyama, T. Takeuchi, T. Mukawa, H. Asanuma Molecular Imprinting. From Fuadamentals to Applications, WILEY-VCH, Weinheim, 2003.

[7] M. L. Kobaishi et al. Adsorption (2007) 13: 315-321

[8] Woo-Sang Lee, Analytical Sciences September 2005, VOL. 21.

[9] K. Farrington, F. Regan, Analytica Chimica Acta 566 (2006) 60-68.

[10] M. Yan, O. Ramström Molecularly Imprinted Materials. Science and Technology, Marcel Dekker, New York, 2005.

[11] K. Farrington, F. Regan Biosensors and Bioelectronics 22 (2007) 1138-1146.

[12] N. Masquè, R. M. Marcè, F. Borrull Trends in Analytical Chemistry 20 (2001) 477-486.

[13] F.G. Tamayo, E. Turiel, A. Martín-Esteban Journal of Chromatography A 1152 (2007) 32-40.

[14] A. McCluskey, C.I. Holdsworth, M.C. Bowyer Organic \& Biomolecular Chemistry 5 (2007) 3233-3244

[15] Y. Fan, Y. Feng, J. Zhang, S. Da, M. Zhang Journal of Chromatography A 1074 (2005) 9-16.

[16] D. Cunliffe, A. Kirby, C. Alexander Advanced Drug Delivery Reviews 5712 (2005) 1836-53.

[17] J. Haginaka, H. Sanbe Journal of Chromatography A 913 (2001) 141-146.

[18] J.Svenson, Analytica Chimica Acta 435 92001) 19-24

[19] G. Bunte et al. Analytica Chimica Acta 591 (2007) 49-56.

[20]Socrates G. "Infrared characteristics group frequencies". Chichester: John Willey E Sons Ltd; 1980, p. 139.

[21]Silverstein, Robert Milton. "Spectrometric identification of organic compounds" John Willey E Sons Ltd. 2005.
[22] R. Ma et al. Journal of Physical Chemistry A 2009, $113,1250-1254$.

[23] J. Clarkson, Journal of Molecular Structure 655 (2003) 413-422.

[24] Ch. Wu, AAPS PharmSciTech 2001; 2 (4) article 24. 